\title{
Exploring Different Stages of Alzheimer's Disease through Topological Analysis of Differentially Expressed Genetic Networks
}

\author{
Aurpan Majumder and Mrityunjay Sarkar
}

\begin{abstract}
Inter-organ dependency of different regions/genes in an eukaryote is nowadays a most challenging issue for researchers. Some neurodegenerative disorders (such as Alzheimer's Disease (A.D)) in a particular organ (Brain) show differential expression patterns across different regions and they are affected by one another. In this work, we have proposed an extended version of an existing concept (Topological Overlap (T.O)). Here we have gone through a "weighted T.O" analysis approach which shows interdependency of all genes present in a network unlike "unweighted T.O" which shows gene dependency of only some significantly connected genes in a network. The weighted as well as un-weighted counterpart helps us to understand the dependency of genes which are responsible for generation and spreading of A. D. Finally, we have ranked the genes (Differentially expressed across different stages of AD) upon the T.O value based permutation test/' $t$-test' and crosschecked the ranked results with a recently published novel ranking scheme. We have also checked the significance of top 15 genes from both methods. In result section we have shown that our method is better than existing one.
\end{abstract}

Index Terms-Alzheimer's disease, G. O., KEGG pathways, topological overlap, weighted and un-weighted measure.

\section{INTRODUCTION}

Alzheimer's disease (AD) is the most common form of dementia. It is an irreversible, progressive brain disease which is characterized by the development of amyloid plaques and neurofibrillary tangles, leading to the loss of connections between nerve cells, or neurons, in the brain, and the death of these nerve cells. With aging this problem becomes worse. In our work we check how genes affect human brain functionality upon aging that cause spreading of $\mathrm{AD}$ from one stage to another.

The rest of the paper is as follow. In next section we have given a brief Theory and literature review. Next we have discussed about the Methodology Then Results and Discussion with respect to other existing method is discussed. This paper concludes with Conclusion and Future work.

\section{THEORY}

A.D is of two types: early-onset and late-onset. Early-

Manuscript received November 4, 2013; revised January 7, 2014.

A. Majumder is with the Department of Electronics and Communication Engineering, N.I.T Durgapur, 713209 India (e-mail: aurpan.nitd@gmail.com).

M. Sarkar is with the Department of Electronics and Communication Engineering, D.I.A.T.M Durgapur, 713212 India (e-mail: mrityu1488@gmail.com). onset Alzheimer's disease occurs in people aged between 30 and 60 years. It is rare, representing less than 5 percent of all people who suffer from this disease. But most cases of Alzheimer's are the late-onset form, which develops after 65 years of age [1], [2]. Many factors are responsible for Alzheimer. Some researchers have suggested that it can be due to calcium-dependent $\mathrm{K}+$ channels in platelets [3] while Lanne has given the concept the ryanodine receptors (RyRs) which is the major intracellular $\mathrm{Ca}^{2+}$ release channel [4] as a probable cause. However diets (proteins) [5], [6] and hormones may also play a pivotal role [7]-[10].

Together all these reasons cause neurodegenerative disease such as Alzheimer. In our work we have found genes responsible for the process that modulates the membrane potential involved in the propagation of a signal in a neuron (Results and discussion).This work also focuses on genes responsible for moving extracellular fluids to and from tissue within a multicellular organism which validates the reason of expansion of the Cerebral Spinal Fluid(CSF) space reducing the CSF turnover rate, thus compromising the CSF sink action to clear harmful metabolites (e.g., amyloid) from the Central Nervous System. Dwindling CSF dynamics greatly harms the interstitial environment of neurons (occurs mostly with aging) [11].

Alzheimer as discussed previously is more susceptible to elderly people. In brain it does not affect different regions simultaneously. AD progresses in stages and is described in terms of incipient (Braak stages III-IV), mild/moderate (Braak stages IV-V) and severe AD (Braak stages V-VI) [12]. While some previous works are focused on genes that have differential topology in gene co expression networks corresponding to different brain regions and to observe the difference in $\mathrm{AD}$ severity across regions [13], our objective is to find genes that have differential topology in gene co expression networks corresponding to different stages of $\mathrm{AD}$ progress and to find out genes/pathways which are responsible for $\mathrm{AD}$ spreading.

\section{Methodology}

\section{A. Network Construction}

We use the method proposed by Ruan and Zhang [14] for network construction where adjacency measure between a pair of genes is being judged through Pearson's correlation. In the beginning we are assuming that it is a fully connected network where the nodes are genes and connection-strength is the weight of edges.

We are evaluating the Topological Overlap

(T.O.) 
between two networks for both weighted and unweighted versions. The corresponding flowchart of the entire process is given in Fig. 1.

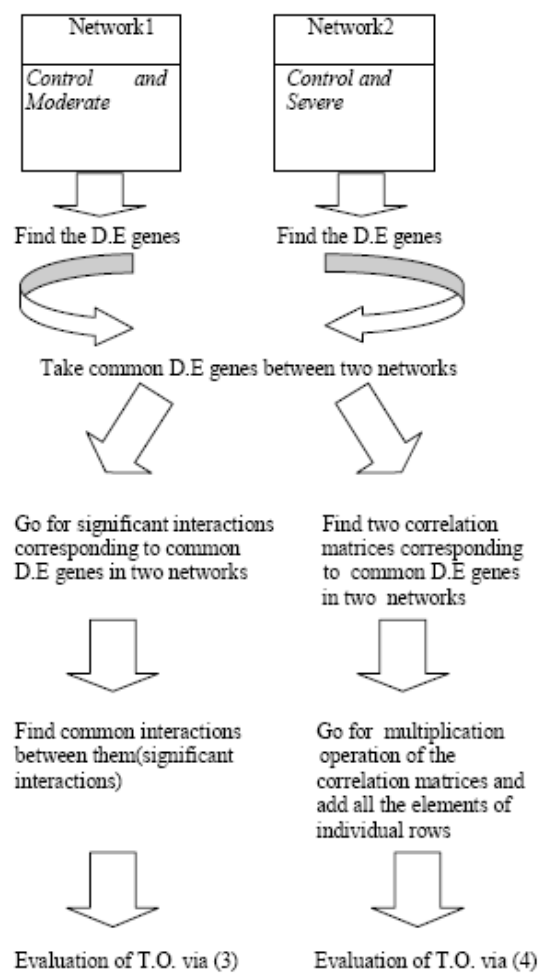

Fig. 1. Flowchart of weighted and unweighted T.O. analysis.

As shown in the flow chart (Fig. 1) we have taken two networks each of them having two sub-networks. Genes in both of the networks are common, but alter in expression levels. In the first network they are control (not affected by A.D) and moderately affected. In the second network they are control and severally affected.

Next we find Differentially Expressed (D.E) genes in the First network (Differentially Expressed between Control and Moderate) and D.E genes in the second network (Differentially Expressed between Control and Severe). Finally we come up with common D.E genes between two networks similar to [13].

\section{B. Unweighted T.O. Analysis}

After finding the common D.E genes between two networks we are taking help of PCIT [15] analysis to find out significant interaction(s) between every pair of genes. In Fig. 1 after taking the common D.E genes the left branch of the tree corresponds to unweighted T.O measure

PCIT: Let there be 3 genes $x, y, z$. Amongst these genes let $x$ and $y$ be connected directly and they are connected via $z$ too. So in between 2 genes there is direct physical gene interaction and via $3^{\text {rd }}$ gene has got indirect physical gene interaction. By this method which gene interaction is direct is done by 2 steps:

1) Partial correlations: For every trio of genes in $x, y$ and $z$, the three first order partial correlation coefficients are computed by:

$$
r_{x y, z}=\frac{r_{x y}-r_{x z} r_{y z}}{\left[\left(1-r_{x z}^{2}\right)\left(1-r_{y z}^{2}\right)\right]^{1 / 2}}
$$

and similarly for $\boldsymbol{r}_{x z, y}$ and $\boldsymbol{r}_{y z, x}$

The partial correlation between $x$ and $y$ given $z$ (here denoted by $\boldsymbol{r}_{x y, z}$ ) indicates the strength of the linear relationship between $x$ and $y$ that is independent (uncorrelated with) of $z$

2) Information theory: This is invoked from the concept of DPI (Data Processing Inequality) [16].

We get the tolerance level/threshold for each association

$$
\varepsilon=\left(\frac{r_{x y, z}}{r_{x y}}+\frac{r_{x z, y}}{r_{x z}}+\frac{r_{y z, x}}{r_{y z}}\right)
$$

After applying the PCIT algorithm we get corresponding to every gene which other genes (columns) have got significant interactions.

Let $\boldsymbol{a}$ be a matrix whose entries signify interactions. After the application of PCIT if the interaction between $i$ th row and $\boldsymbol{j}$ th column is significant then the corresponding entry $\boldsymbol{a}_{i j}=\mathbf{1}$ else $\boldsymbol{a}_{i j}=\mathbf{0}$. In this way two matrices are formed for the two networks. Next we explore which interactions are significant and common between two networks for each gene. Let for gene $\boldsymbol{i}$ in network $1 X$ no of interaction(s) is/are significant, and in network 2 it is $Y$. So as in [13] the T.O. of gene $i$ between two networks can be defined as

$$
\text { T.O. }_{i}=\frac{X \cap Y}{\max (X, Y)}
$$

\section{Weighted T.O. Analysis}

Unlike unweighted concept here we retain with the correlation values between each pair of common D.E genes for the two networks. This is followed by our multiplication operation of the two correlated matrices. Finally we add up the gene-gene interactions per gene to determine the corresponding gene's proportional T.O significance. In Fig. 1 after taking the common D.E genes the right branch of the tree corresponds to weighted T.O measure.

Here, we found the significance of gene $i$ in both networks after conducting the multiplication and addition operations of the two correlated matrices. Hence, the $i$ 'th entry possessing a high value highlights that the correlative significance of $\boldsymbol{i}$ 'th gene with other genes in both networks is very strong whereas if the $\boldsymbol{i}$ 'th entry has got a low value, it clarifies that the correlative significance of $\boldsymbol{i}$ 'th gene with the other genes is quite weak.

After multiplication (of two matrices) and addition the $n \times 1$ ( $n=$ total number of common D.E. genes) matrix is stored in $A$. Next, we add up the elements of individual rows in both matrices separately and the $n \times 1$ matrices thus formed are stored in $B$ and $C$. So the T.O. of gene $i$ between two networks can be defined as:

$$
\text { T.O. } i=\frac{\min (A)}{\max \left(B_{i}, C_{i}\right)}
$$

Here $B_{i}$ and $C_{i}$ represents the $i$ 'th element of matrix $B$ and $C$ 
After getting the T.O value for each common D.E. gene we go for the permutation test [17]. Depending upon the result of the permutation test we proceed for ranking.

\section{Pre-Processing}

We have taken the dataset from publicly available database [18]. As the size of the dataset is very high $(54,675$ genes with 30 time instants), so the time as well as space complexity is very high if we are about to analyze the entire dataset. For this reason before going for detailed analysis we have to go for some pre-processing. There are two stages, they are

1) The skewness of the dataset is high, for different entries. To remove the skewness we go for 'lognormal' distribution. This makes the distribution of the dataset somehow uniformly distributed.

2) In the second stage we evaluate the standard-deviation of all genes over different expression levels. Setting a threshold value we fetch the expression values of the corresponding genes which give a standard-deviation greater than the threshold.

\section{RESUlTS AND DisCUSSION}

As mentioned in the previous section the original dataset has 54,675 entries. After applying the pre-processing strategies the total entries get reduced to 10,000 (We are choosing top 10,000 genes). We have restricted our work between control and moderate and control and severe. We are not taking incipient stage into our account because the change of expression levels between control and incipient is not so prominent.

First, we had to find the D.E genes between control and moderate and control and severe. We have done this by
DEGseq (R) [19] (It is a package to find the Differentially Expressed genes from the gene expression value itself. Here depending upon expression values of genes at different time instants and by a particular threshold ' $p$-value'/' $z$-score'/' $q$ value' D.E genes are computed). This validation/significance of our method has been done through the following steps:

1) We have searched the significance of the T.O values for all the 66 common D.E.genes (for both weighted and unweighted measure) by random permutation test/'t'-test.

2) Based upon the result of the permutation test/' $t$ '-test the 66 common D.E. genes are ranked

3) We have also computed the ranking of 66 common D.E.genes by Diffrank [20].

4) Next we have fetched top 15 ranked genes(approximately top $25 \%$ of common D.E. genes) (from both weighted and unweighted measure) computed by proposed one as well as by Diffrank.

After fetching the top 15 genes we have found that the number of common genes between proposed method and Diffrank are very low. Out of top15 genes in weighted measure only 4 genes are common and in unweighted measure only 3 genes are common.

5) Next we have found GO term based significance as well as pathways based significance of the top 15 ranked genes by [21], [22].

In Tables I, II, III, and IV we have enlisted significant G.O. terms and KEGG pathways along with ' $p$-value' and number of O.R.F name of the genes associated with them. Here we have found that out of the 15 top D.E genes most of them have associated differently to form different G.O. terms as well as pathways.

TABLE I: Significant Go TeRms in PRoposed Method as Well as in DifFRanK by Weighted Measure

\begin{tabular}{|c|c|c|c|c|c|}
\hline \multicolumn{3}{|c|}{ Proposed Algorithm } & \multicolumn{3}{|c|}{ Diffrank } \\
\hline GO-Terms & 'p-value' & Genes & GO-Terms & 'p-value' & Genes \\
\hline GO:0046885 & 0.0346 & $\begin{array}{l}2 \\
\text { trerf1, } \text { kynu }\end{array}$ & GO:0006569 & 0.0348 & $\begin{array}{l}3 \\
\text { mmp10, kynu, kcne1 }\end{array}$ \\
\hline GO:0032350 & 0.0346 & $\begin{array}{l}3 \\
\text { kynu, usp28, itgb3 }\end{array}$ & G0:0046218 & 0.0348 & $\begin{array}{l}2 \\
k y n u, j a k 1\end{array}$ \\
\hline GO:0006569 & 0.0349 & $\begin{array}{l}2 \\
\text { jak1, efcab2 }\end{array}$ & GO:0002070 & 0.0352 & $\begin{array}{l}2 \\
\operatorname{cog} 3, \operatorname{ciaol} 1\end{array}$ \\
\hline GO:0046218 & 0.0349 & $\begin{array}{l}2 \\
\operatorname{cog} 3, \boldsymbol{e f c a b 2}\end{array}$ & GO:0005131 & 0.0359 & $\begin{array}{l}1 \\
\text { efcab2 }\end{array}$ \\
\hline GO:0004718 & 0.0352 & $\begin{array}{l}1 \\
\text { fancd } 2\end{array}$ & GO:0002064 & 0.0359 & $\begin{array}{l}1 \\
\text { arhgap24 }\end{array}$ \\
\hline
\end{tabular}

TABLE II: SignifiCANT Go TERMS In PROPOSED METHOD AS WELl AS IN DifFRANK By UNWEIGHTED MEASURE

\begin{tabular}{|c|c|c|c|c|c|}
\hline \multicolumn{3}{|c|}{ Proposed Algorithm } & \multicolumn{3}{|c|}{ Diffrank } \\
\hline GO-Terms & 'p-value' & Genes & GO-Terms & 'p-value' & Genes \\
\hline GO:0015459 & 0.00095 & $\begin{array}{l}2 \\
\text { kcne1, mmp10 }\end{array}$ & GO:0030303 & 0.019 & $\begin{array}{l}2 \\
\text { ciaol, } \mathbf{m m p 1 0}\end{array}$ \\
\hline GO:0016247 & 0.00162 & $\begin{array}{l}2 \\
t p 73, k c n m b 2\end{array}$ & GO:0018676 & 0.019 & $\begin{array}{l}2 \\
\operatorname{cyp} 2 c 9, \text { itgb3 }\end{array}$ \\
\hline GO:0015457 & 0.00162 & $\begin{array}{l}3 \\
\text { ciao1, kcne1, mmp10 }\end{array}$ & GO:0036767 & 0.019 & $\begin{array}{l}2 \\
\text { kcnmb2, mmp10 }\end{array}$ \\
\hline GO:0030303 & 0.0093 & $\begin{array}{l}3 \\
\text { trim14, tp73, } c d c 20 b\end{array}$ & GO:0019113 & 0.0271 & $\begin{array}{l}2 \\
\text { kcnmb2, plxnd1 }\end{array}$ \\
\hline GO:0008076 & 0.0093 & $\begin{array}{l}2 \\
\text { mmp10, } k c n m b 2\end{array}$ & GO:0018675 & 0.0274 & $\begin{array}{l}3 \\
v \operatorname{sp53}, \operatorname{tp} 73, \text { itgb3 }\end{array}$ \\
\hline
\end{tabular}




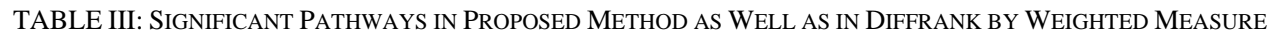

\begin{tabular}{|c|c|c|c|c|c|}
\hline \multicolumn{3}{|l|}{ Proposed Algorithm } & \multicolumn{3}{|l|}{ Diffrank } \\
\hline Pathways & 'p-value' & Genes & Pathways & 'p-value' & Genes \\
\hline Tryptophan metabolism & 0.0220 & $\begin{array}{l}3 \\
\text { kynu, } \operatorname{cog} 3, \text { efcab2 }\end{array}$ & $\begin{array}{l}\text { Jak-STAT signalling } \\
\text { pathway }\end{array}$ & 0.0212 & $\begin{array}{l}3 \\
\text { jak1, kcne1, trdv3 }\end{array}$ \\
\hline $\begin{array}{l}\text { Pancreatic } \\
\text { cancer }\end{array}$ & 0.0383 & $\begin{array}{l}3 \\
\text { jak1, itgb3, usp } 28\end{array}$ & Tryptophan metabolism & 0.0220 & $\begin{array}{l}3 \\
\text { csh1, kynu, mmp10 }\end{array}$ \\
\hline Leishmaniasis & 0.0399 & $\begin{array}{l}2 \\
\text { usp28, efcab2 }\end{array}$ & Pancreatic cancer & 0.0383 & $\begin{array}{l}2 \\
\text { ciao1, } \operatorname{cog} 3\end{array}$ \\
\hline $\begin{array}{l}\text { Arrhythmogenic right } \\
\text { ventricular } \\
\text { cardiomyopathy }\end{array}$ & 0.0416 & $\begin{array}{l}2 \\
\text { efcab2, } \operatorname{cog} 3\end{array}$ & Leishmaniasis & 0.0399 & $\begin{array}{l}1 \\
\text { efcab2 }\end{array}$ \\
\hline ECM-receptor interaction & 0.0459 & $\begin{array}{l}2 \\
\text { trerf1, } \text { mgc3771 }\end{array}$ & & & \\
\hline $\begin{array}{l}\text { Hypertrophic } \\
\text { cardiomyopathy }(\mathrm{HCM})\end{array}$ & 0.0464 & $\begin{array}{l}1 \\
\text { c20orf78 }\end{array}$ & & & \\
\hline Hematopoietic cell lineage & 0.0488 & $\begin{array}{l}1 \\
\text { fancd } 2\end{array}$ & & & \\
\hline
\end{tabular}

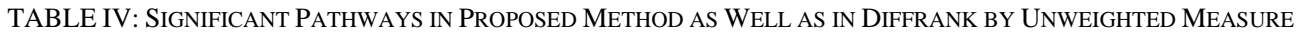

\begin{tabular}{|c|c|c|c|c|c|}
\hline \multicolumn{3}{|l|}{ Proposed Algorithm } & \multicolumn{3}{|l|}{ Diffrank } \\
\hline Pathways & 'p-value' & Genes & Pathways & 'p-value' & Genes \\
\hline p53 signaling pathway & 0.0253 & $\begin{array}{l}3 \\
\text { tp73, cdc20b, mmp10 }\end{array}$ & $\begin{array}{l}\text { Linoleic acid } \\
\text { metabolism }\end{array}$ & 0.0212 & $\begin{array}{l}2 \\
\text { cyp2c9, plxnd1 }\end{array}$ \\
\hline $\begin{array}{l}\text { Vascular smooth muscle } \\
\text { contraction }\end{array}$ & 0.0424 & $\begin{array}{l}2 \\
\text { kcnmb2, } \boldsymbol{e f c a b 2}\end{array}$ & $\begin{array}{l}\text { Arachidonic acid } \\
\text { Metabolism }\end{array}$ & 0.0422 & $\begin{array}{l}2 \\
\text { itgb3, ciaol }\end{array}$ \\
\hline $\begin{array}{l}\text { Neurotrophin signaling } \\
\text { pathway }\end{array}$ & 0.0460 & $\begin{array}{l}2 \\
\text { kcnmb2, trim14 }\end{array}$ & Retinol metabolism & 0.0472 & $\begin{array}{l}1 \\
\text { kcnmb2 }\end{array}$ \\
\hline
\end{tabular}

Top 25\% ranked genes have been selected based upon their low T.O score because our main concern is to select condition specific genes. Thus top 25\% genes sharing less number of neighbours than others (in different conditions) are selected. They have more enriched G.O. terms as well as more enriched biological pathways than the remaining low ranked genes, so to check the effectiveness of the proposed method we have opted to compare top $25 \%$ genes from both methods.

From Tables I, II, III, and IV (List is prepared according to their ' $p$ '-value as well as the impact of these G.O. terms/pathways in A.D. described in different literatures) it is evident that our proposed methodology gives better result than Diffrank both in terms of G.O enrichment analysis as well as of pathway analysis.

Our method shows better result because the existing method [20] focuses on local (differential connectivity which is the local difference between two networks calculated by the number of genes associated with a particular gene) as well as on global concept (between centrality: which calculate the change in the expression levels of central genes), but as given in [13] A.D does not affect all the brain regions at a time but there are differences in $\mathrm{AD}$ severity across regions. So it shows more local phenomena than the global one. In general all the genetic diseases show this kind of pattern. It [20] also shows a problem of controlling the value of the trade-off parameter $(\lambda)$, trying to make up a balance between local and global connectivity. So the optimality of the result is solely dependent on this parameter, making the problem more parameter driven. Here we proposed an extended version of an existing method [13] and we have shown that it outperforms other existing method [20].

In Tables I, and II we have enlisted some significant G.O. terms. Some of the G.O terms found by the proposed method are responsible for generation and spreading of A.D in Human and other primates. They have been verified in some recent literatures like : GO:0046885(regulation of hormone biosynthetic process)[23], GO:0032350(regulation of hormone metabolic process)[24], GO:0006569(tryptophan catabolic process)[25], GO:0046218(indolalkylamine catabolic process)[26], GO:0004718(protein tyrosine kinase activity)[27], GO:0015459(potassium channel regulator activity)[28], GO:0016247(channel regulator activity)[29], GO:0015457(Transport) [30], GO:0008076(voltage-gated potassium channel complex) [31].

Again in Tables III and IV we have listed some significant pathways. Some of them also verified to be responsible for generation and spreading of A.D in some recent literatures like: Tryptophan metabolism and Leishmaniasis [32], Arrhythmogenic right Ventricular cardiomyopathy [33], ECM-receptor interaction [34], Hypertrophic cardiomyopathy [35], Hematopoietic cell lineage [36], p53 signaling pathway [37], Neurotrophin signalling pathway [38], Retinol metabolism [39].

\section{CONCLUSION AND FUTURE WORK}

In this work we have done T.O. analysis from D.E. genes. D.E. genes are found by comparing the gene-expression values between two states/regions. As discussed in the result and discussion section first we have searched for common D.E genes between two networks, this common set of 
genes(D.E) will act differently in the two networks(first network comprises control and moderately affected genes and the second network comprises control and severely affected genes).

Next to find the unweighted T.O. measure we have searched for significant gene to gene interactions (by the common D.E genes) in both networks and then the common interactions between them.

In weighted T.O measure instead of significant gene to gene interactions we have to focus on each and every interaction. So here we have searched for correlation matrices of the common D.E genes in both networks, then we have gone for multiplication and addition operations over the correlation matrices.

Basically here we have developed a new approach to find the T.O value. Other existing method [13] focuses only on unweighted T.O measure which takes into account only of some significant gene to gene interactions. So the overall gene dependency and gene significance in a particular network is not totally highlighted. On the other side weighted T.O measure focuses on each and every interaction how strong or weak it is, and accordingly we can get a clear picture of gene dependency and gene significance in a network. In Tables III, and IV we can observe this where the number of enriched biological pathways in weighted measure is more than the unweighted counterpart.

Here we have also proposed a novel concept of ranking based upon the T.O. measure, which unlike [20] does not depend upon any local or global measure or any trade-off parameter, thus gives a better result than the existing method of ranking [20].

Maintaining this line of thought our next step being finding out D.E genes from gene expression values and phenotype specific traits. In such a context, we are interested to explore this idea through linear and non-linear methodologies. Accordingly, in the linear method we will find the correlation between gene expression values and phenotype specific trait whereas in the non-linear method we will go through mutual information and /or polynomial and spline regression model based measure of dependency between them. This would probably allow us to check the compatibility of trait specific analysis by comparing the result with other existing methods.

\section{REFERENCES}

[1] E. Kensinger, "Early and late onset as subdivisions of Alzheimer's Disease," The Harvard Brain, pp. 26-29, 1996

[2] Alzheimer's Disease Education and Referral centre, National Institute of Aging. (June 2011). [Online]. Available: www.nia.nih.gov/alzheimers/topics/alzheimers-basics

[3] H. A. D. Silva, J. K. Aronson, D. G. G. Smith, K. A. Jobst, A. D. Smith, "Function of potassium channels in platelets of patients with Alzheimer's disease," Lancet, November 1998.

[4] J. T. Lanne, "Ryanodine receptor physiology and its role in disease," Calcium Signaling Advances in Experimental Medicine and Biology, Springer, vol. 740, pp. 217-234, 2012.

[5] S. Seneff, G. Wainwright, and L. Mascitelli, "Nutrition and Alzheimer's disease: The detrimental role of a high carbohydrate diet," European Journal of Internal Medicine, December 2010.

[6] D. J. Selkoe, "Alzheimer's disease: Genes, proteins, and therapy," Physiological Reviewes, vol. 81, no. 2, April 2001.

[7] N. Bogdanovic et al,. "On the turnover of brain cholesterol in patients with Alzheimer's disease. Abnormal inductionthe cholesterolcatabolic enzyme CYP46 in glial cells," Neurosci. Lett. 314, pp. 4548, [PMID: 11698143], 2001.
[8] N. Gustin, "Researchers discover link between insulin and Alzheimer's," American Association for the Advancement of Science, March 2005.

[9] A. Morinaga, K. Ono, J. Takasaki, T. Ikeda, M. Hirohata, and M. Yamada, "Effects of sex hormones on Alzheimer's disease-associated $\beta$-amyloid oligomer formation in vitro," Exp Neurol, April 2011.

[10] V. Hosur, R. H. Loring, “ $\alpha_{4} \beta_{2}$ Nicotinic receptors partially mediate anti-inflammatory effects through janus kinase 2-signal transducer," Mol Pharm, October 2010.

[11] C. E. Johanson, J. A. Duncan, P. M. Klinge, T. Brinker, E. G. Stopa and G. D. Silverberg, "Multiplicity of cerebrospinal fluid functions: New challenges in health and disease," Cerebrospinal Fluid Research, pp. 5-10, May 2008.

[12] H. Braak and E. Braak, "Neuropathological staging of Alzheimerrelated changes," Acta Neuropathologica, vol. 82, pp. 239-259, June 1991.

[13] M. Ray and W. X. Zhang: “Analysis of Alzheimer's disease severity across brain regions by topological analysis of gene co-expression networks," BMC Systems Biology, vol. 4, pp. 136, October 2010.

[14] J. Ruan, A. K. Dean, and W. Zhang, "A general co-expression network-based approach to gene expression analysis: comparison and applications," BMC Systems Biology, vol. 4, no. 8, February 2010.

[15] A. Revarter and E. K. Chan, "Combining partial correlation and an information theory approach to the reversed engineering of gene coexpression networks," Bioinformatics, vol. 24, no. 21, pp. 2491-2497, November 2008

[16] J. B. Normand, "An intuitive proof of the data processing inequality," Quantum Inf. Comput., vol. 12, no. 5\&6, pp. 432-441, July 2012.

[17] M. Ojala and G. C. Garriga, "Permutation tests for studying classifier performance," Journal of Machine Learning Research, pp. 18331863 , June 2010.

[18] Gene Expression Omnibus, [Online]. Available: http://www.ncbi.nlm.nih.gov/geo:Nucleic Acid Res:2005Jan1;Database Issue:D562-6

[19] L. Wang, Z. Fenq, X. Wang, X. Wang, and X. Zhang, "DEGseq: An $\mathrm{R}$ package for identifying differentially expressed genes from RNAseq date," Bioinformatics, vol. 26, no. 1, pp. 136-144, January 2010.

[20] O. Odibat and C. K. Reddy, "Ranking differential hubs in gene coexpression networks," Journal of Bioinformatics and Computational Biology, vol. 10, no. 1, January 2012.

[21] GOstat. (October, 2013). [Online]. Available: http://www.gostat.wehi.edu.au

[22] GeneTrail. (October, 2013). [Online]. Available: http://www.genetrail.bioinf.uni-sb.de/

[23] L. Jing et al., "Genetic ablation of luteinizing hormone receptor improves the amyloid pathology in a mouse model of Alzheimer disease," J. Neuropathol Exp Neurol, vol. 69, no. 3, pp. 253-261, PubMed, March 2010.

[24] M. M. Hasan, "Comparative systems-level analysis of the G1/S transition in yeast and higher eukaryotes: focusing on the Whi5/Rb network and initiation of DNA replication," Ph.D. Dissertation, Department of Biotechnology and Biosciences, University of MilanBicocca, Italy, December 2011.

[25] A. Kowarsch et al., "Knowledge-Based matrix factorization temporally resolves the cellular responses to IL-6 stimulation," Biomed Central, vol. 11, pp. 585, November 2010.

[26] K. James, "Knowledge derivation and data mining strategies for probabilistic functional integrated networks," Ph.D. Thesis, School of Computing Science, Newcastle University, U.K., July 2011.

[27] X. Z. Han, T. Shen, and H. X. Lou, "Dietary polyphenols and their biological significance," International Journal of Molecular Sciences, pp. 950-988, September 2007.

[28] K. S. Lynn, C. H. Lu, H. Y. Yang, W. L. Hsu, and W. H. Pan, "Construction of gene clusters resembling genetic causal mechanisms for common complex disease with an application to young-onset hypertension," BMC genomics, pp. 14-497, July 2013.

[29] K. Bossers et al., "Concerted changes in transcripts in the prefrontal cortex precede neuropathology in Alzheimer's disease," Brain, vol. 133, pp. 3699-3673, July 2013.

[30] A. B. Goodman and A. B. Pardee, "Evidence for defective retinoid transport and function in late onset Alzheimer's disease," PNAS, pp. 2901-2905, December 2002.

[31] Y. Pertzov et al., "Binding deficits in memory following medial temporal lobe damage in patients with voltage-gated potassium channel complex antibody-associated limbic encephalitis," Brain ,vol. 136, no. 8, pp. 2474-2485, August 2013.

[32] M. P. Barrett, R. J. S. Burchmore, and A. Stich, "Role of heme and heme-proteins in trypanosomatid essential metabolic pathways," Lancet, vol. 362, no. 9394, pp. 1469-1480, February 2003. 
[33] NCBI. [Online]. Available: http://www.ncbi.nlm.nih.gov /IEB/Research/Acembly

[34] B. W. Kunkle et al., "Whole-Exome sequencing in early-onset Alzheimer disease families identifies rare variants in multiple Alzheimer-related genes and processes," Alzheimer's Disease Genetics Consortium, 2013.

[35] T. Force, K. Kuida, M. Numchuk, K. Parang, and J. M. Kyriakis: "Inhibitors of protein kinase signaling pathways," American Heart Association, vol. 109, pp. 1196-1205, March 2004.

[36] M. Squillario and A. Barla, "A computational procedure for functional characterization of potential marker genes from molecular data: Alzheimer's as a case study," BMC Medical Genomics, vol. 4, no. 55, July 2011.

[37] A. V. Gudkov and E. A. Kamarova, "Pathologies associated with the p53 Response," Cold Spring Harbor Perspectives in Biology, vol. 2, no. 4, pp. 503-516, April 2010.

[38] A. L. Torre et al., "A role for the tyrosine kinase ACK1 in neurotrophin signaling and neuronal extension and branching," Cell Death and Research, vol. 4, no. 602, April 2013.

[39] M. A. Lane and S. J. Bailey, "Role of retinoid signalling in the adult brain," ELSEVIER: Progress in neurobiology, vol. 75, pp. 275-293, March 2005.

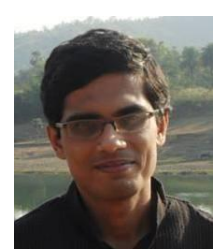

Aurpan Majumder had obtained his B.Tech. in 2002 in electronics \& telecommunication Engg. from Haldia Institute of Technology, India and M.Tech. in 2005 in computer science from Jadavpur University, India. Currently, he is working as an assistant professor at National Institute of Technology, Durgapur, India. He is pursuing $\mathrm{PhD}$ in the domain of gene differential coexpression analysis. His research interests also include biomedical signal and image processing.

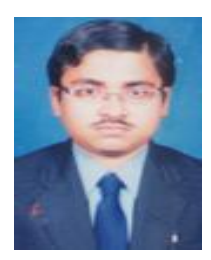

Mrityunjay Sarkar had obtained his B.Tech. in electronics and communication, Eng. from Asansol Eng. College and M.Tech. in telecommunication engineering from National Institute of Technology Durgapur. He is now working as an asst. prof. in Durgapur Institute of Advanced Technology and Management, Durgapur in the department of Electronics and Communication Eng. His research areas of interest being Image Processing, Soft Computing, Gene CoExpression Network Analysis. 